\title{
Assessing adherence to toothbrushing instructions
}

\author{
Assessing adherence with toothbrushing instructions using a data logger toothbrush G. McCracken, J. Janssen, \\ L. Heasman, F. Stacey, N. Steen, M. deJager and P. Heasman Br Dent J 2005; 198: 29-32
}

\section{Objectives}

To evaluate patient compliance with toothbrushing instructions using a data logger and a brushing diary.

\section{Methods}

Dental patients were provided with powered toothbrushes modified to carry electronic data loggers. Demonstration of the most effective way to use the toothbrush was provided with instructions to brush for 2 minutes morning and evening. Data logger brushes were supplied for two episodes of 8 weeks. Brushing time was also recorded in a diary during episode 1.

Results

Data from 14 brushes (2,287 recordings) were used to evaluate compliance in episode 1 . Nine brushes (1,526 recordings) were used in episode 2 . A total of 2,333 brushing events were reported manually in the diaries. Data logger records during episode 1 showed that only $34 \%$ of events were compliant with the instruction of a 2 minute brushing time, 20\% were partially compliant (within \pm 30 s of 2 minutes), and $46 \%$ of events were non-compliant ( $>30$ s from 2 minutes). The respective proportions for episode 2 were $24 \%, 24 \%$ and $52 \%$. Diary data reported $58 \%$ of events as compliant with 42\% non-compliant.

\section{Conclusions}

Manually completed brushing diaries do not provide an accurate reflection of subjects' compliance with toothbrushing instructions.

\section{IN BRIEF}

- Electronic data logging verified the level of compliance with toothbrushing regimens.

- Large differences were observed between recorded (electronic loggers) and reported (diaries) levels of compliance.

- Clinicians should always take account of issues of compliance relating to the success or failure of any clinical intervention or therapy.

\section{COMMENT}

Compliance with self-care is vital to the success of dental treatment and is thus important to all dental professionals. It is a difficult field of study, as self-reporting of compliance has been shown to be unreliable. The use of data loggers in this work provides a novel means of investigation of compliance and this clearly written, logical and relevant paper succeeds in addressing this important issue.

Data loggers were placed in powered toothbrushes and recipients also received standardised toothbrushing instructions. On each brushing occasion the data loggers recorded brushing time, brushing force and re-charge time between sessions.

The experiment lasted for 8 months:

- In the first two, both data loggers and a brushing diary were used

- In the next four months a powered brush minus data logger was used

- In the final two months, data loggers but no brushing diaries were used.

Results from the first two months showed $34 \%$ of brush uses complied, $20 \%$ partially complied and $46 \%$ were non-compliant with instruction. Corresponding figures for the final two months were $24 \%, 24 \%$ and $52 \%$. Diary data reported $58 \%$ of events as compliant and $42 \%$ non-compliant - which clearly differs from the recorded data. It confirms the differences, which may be observed by many dentists, between reported patient self-care and the clinical evidence found on examination.

The paper found that participants could be divided into three almost equal categories: one-third spending less time than advised, one-third following advice and one-third exceeding the time requested. This is significant as it suggests that a substantial proportion of patients do not comply with self-care instructions (even over a short period of time) in spite of the fact that they have disease which is likely to be successfully managed only if selfcare advice is followed. Medico-legally this is relevant, and suggests that dentists should ensure that they have a record of the self-care advice they have given to patients. Clearly, lack of compliance may adversely affect treatment results and future prognosis.

The numbers involved in this study are small and it is not possible to extrapolate the results directly to a large population, but the information gained is a very useful indicator of the degree of the compliance problem facing clinicians. This work is of value to every practising dentist.

\section{A. D. Gilbert, Senior Lecturer in Periodontology,} University of Dundee doi: $10.1038 / s j . b d j .4811967$ 